\title{
Study Of The Association Of Tumor Deposits With Tumour-infiltrating Lymphocytes And Prognosis In Gastric Cancer Patients
}

\section{Xinyue Li}

First Affiliated Hospital of Jinzhou Medical University

Jing Yang ( $\sim$ jydyj781125@163.com )

First Affiliated Hospital of Jinzhou Medical University

\section{Research Article}

Keywords: tumour deposits, tumour-infiltrating lymphocytes, prognosis

Posted Date: August 3rd, 2021

DOl: https://doi.org/10.21203/rs.3.rs-754314/v1

License: (c) (1) This work is licensed under a Creative Commons Attribution 4.0 International License.

Read Full License 


\section{Abstract}

Background: To investigate the relationship between tumour deposits(TDs) with the clinicopathological

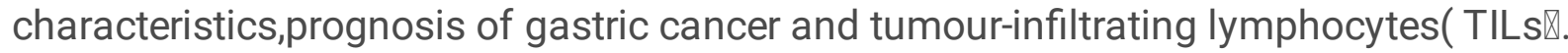

Methods: The pathological findings of 369 patients with gastric cancer were retrospectively analysed to observe the expression of TDs, and the levels of stromal TILs. The relationships between TDs status, clinicopathological characteristics, and TILs infiltration level were compared using the chi-square test, and rank data were tested using the rank sum test. Kaplan-Meier was used for survival analysis, and the log-rank test was used to determine the differences in survival curves between groups. The prognostic value of TDs was assessed using multivariate Cox proportional hazards regression analysis.

Results: TDs were significantly associated with sex, Lymphovascular invasion, Perineural invasion, pathological TNM stage, and clinical stage (all P<0.05). TILs levels were lower in TDs $(+)$ group and higher in TDs(-) group. TDs(+) group had poor Disease-free survival, cancer-specific survival , and overall survival as compared with TDs(-) groups.

Conclusions: TDs is negatively correlated with TILs, and TDs+ was an Independent predictors of the prognosis of gastric cancer.

\section{Introduction}

Gastric cancer is an important cancer worldwide, with the fourth highest mortality rate [1]. At present, the prognosis of gastric cancer patients and the formulation of treatment plans mainly rely on clinicopathological parameters and molecular indicators [2, 3], but due to the heterogeneity of gastric cancer, none of these indicators can fully accurately reflect the prognosis of gastric cancer patients. Thus, it is especially important to study and enrich new prognostic indicators. Tumour deposits (TDs) were first described as colorectal cancer mesenteric satellites in 1935 [4], and according to the American Joint Cancer Committee (AJCC) TNM staging system (8th ed.) for colorectal cancer, TDs were clearly defined as discrete tumour nodules within the lymphatic drainage area of primary cancer and without identifiable lymph node tissue or identifiable vascular or neural structures [5]. In colorectal cancer, TDs are included in staging treatment because they have been shown to be an independent prognostic factor [6, 7]. However, TDs have not been included in the pathological staging of gastric cancer due to limited research evidence. Only a few studies in the literature have found TDs to be strongly associated with a poor prognosis in gastric cancer $[2,8,9]$. In addition, tumor immune response has gradually become a hot issue in recent years as the study of tumor microenvironment has been intensified. However, the relationship between tumor deposition and tumor microenvironment has been rarely reported,tumorinfiltrating lymphocytes, as an important component of tumor microenvironment, are an important mechanism for the body to cope with tumor cells and induce tumor immune response [10,11]. Therefore, this study aimed to investigate the relationship between TDs and TILs and prognosis, and provide new ideas for the diagnosis and treatment of gastric cancer. 


\section{Materials And Methods}

\section{Clinical information}

Clinicopathological data were collected from January 2016 to December 2019 from patients who underwent surgical resection of gastric cancer at The First Affiliated Hospital of Jinzhou Medical University. The inclusion criteria were as follows: (1) patients with pathologically confirmed gastric cancer; (2) patients who had not received preoperative neoadjuvant radiotherapy or other adjuvant treatment;(3) patients with complete clinical information and follow-up information. The exclusion criteria were as follows: (1) patients lost to follow-up; (2) those with other malignancies; (3) those with a preoperative co-infection or autoimmune disease. This study was approved by the Ethics Committee of The First Affiliated Hospital of Jinzhou Medical University (ethics number: KYLL 202029).

2. Interpretation of TDs

Two independent pathologists separately reviewed pathological sections of gastric cancer using a double-blind method, and disagreements were confirmed by a third expert.Positive TD(TDs+) is defined as discrete tumor nodules within the lymph drainage area of the primary carcinoma without identifiable lymph node tissue or identifiable vascular or neural structure. [5].

\section{Interpretation of TILs}

The two independent pathologists assessed the percentage of stromal TILs in the central tumor and invasive margin of gastric cancer foci using a double-blind method and the assessment methods recommended by the 2014 International Working Group on Tumour-Infiltrating Lymphocytes [12]. The methods for interpretation were as follows: (1) determine the extent of TIL assessment (TILs within the tumour border, including border locations, were assessed, and extratumoural and intraepithelial TILs, peritumoral tertiary lymphatic structures, and extensive necrosis or fibrosis were not assessed); (2) target the area of stromal TILs in the tumour (intraepithelial TILs were not assessed); (3) scan the entire field of view at low magnification; (4) determine the type of infiltrating cells, and count only single nucleated cells (lymphocytes and plasma cells); and (5) derive a percentage based on the ratio of the area occupied by stromal TILs to the total area of the interstitium, from which two groups were classified: a group with a low-to-medium TILs, with a percentage of $<40 \%$, and a group with a high TILs, with a percentage of $40-$ $90 \%$. Averages were taken after the evaluation of multiview observations and were not focused on the hotspot view with the most infiltration.

\section{Follow-up information}

Follow-up started on the first day after surgery, and the median follow-up time was 27 (1-54) mon, with reviews per month for 3 y post-surgery, every 6 mon for 3- 5 y post-surgery, and every 12 mon after 5 years post-surgery. Follow-up information was obtained by telephonic follow-up. Disease-free survival (DFS) was defined as the time from the start of follow-up until disease recurrence, metastasis, or progression; cancer-specific survival (CSS) was defined as the time from the start of follow-up to death 
due to gastric cancer; and overall survival (OS) was defined as the time from the start of follow-up to the patient's death due to any other cause.

\section{Statistical processing}

All data were statistically analysed using SPSS 21.0 software (IBM Corporation, Armonk, NY, USA). The relationships between TDs status, with clinicopathological characteristics, and TILs infiltration level were compared using the chi-square test, and rank data were tested using the rank sum test. Kaplan-Meier was used for survival analysis, and the log-rank test was used to determine the differences in survival curves between groups. The prognostic value of TDs was assessed using multivariate Cox proportional hazards regression analysis. $P<0.05$ indicated a statistically significant difference.

\section{Results}

1. Relationships between tumour deposits and the clinicopathological characteristics

Of the 369 gastric cancer cases, 81 had TDs+ (22.0\%) (Fig. 1). TDs were significantly associated with sex, Lymphovascular invasion(LVI), Perineural invasion(PNI), pathological TNM stage, and clinical stage (all $\mathrm{P}<0.05)$, whereas there was no statistical difference between age, Histologic grade, Lauren's classification, and mismatch repair gene(MMR) (all $P>0.05)$ (Table 1). For further analysis, the optimal cut-off values for the number and maximum diameter of TDs were selected based on the receiver operating characteristic curve (ROC), and the samples were divided into the number of TDs $(<4$ and $\geq 4)$ and the maximum diameter of TDs $(<7 \mathrm{~mm}$ and $\geq 7 \mathrm{~mm}$ ). The number of TDs was significantly associated with pathological $\mathrm{N}$ stage $(\mathrm{P}<0.05)$. The maximum diameter of TDs was significantly correlated with Lauren's classification $(P<0.05)$. 
Table 1

Association between tumor deposits clinicopathologic characteristics of gastric cancer.

\begin{tabular}{|c|c|c|c|}
\hline & TDs+ & TDs- & $\mathbf{P}$ \\
\hline Sex & 65 & 196 & 0.033 \\
\hline Male & 16 & 92 & \\
\hline \multicolumn{4}{|l|}{ Female } \\
\hline Age,years & 27 & 98 & 0.907 \\
\hline$\leq 60$ & 54 & 190 & \\
\hline \multicolumn{4}{|l|}{$\bigotimes 60$} \\
\hline Histologic grade & 69 & 224 & 0.145 \\
\hline Undifferentiated & 12 & 64 & \\
\hline \multicolumn{4}{|l|}{ Differentiated } \\
\hline Lauren's classification & 41 & 152 & 0.241 \\
\hline diffuse type & 27 & 72 & \\
\hline mixed type & 13 & 64 & \\
\hline \multicolumn{4}{|l|}{ intestinestype } \\
\hline LVI & 77 & 171 & 0.000 \\
\hline Yes & 4 & 117 & \\
\hline \multicolumn{4}{|l|}{ No } \\
\hline PNI & 71 & 152 & 0.000 \\
\hline Yes & 10 & 136 & \\
\hline \multicolumn{4}{|l|}{ No } \\
\hline MMR & 76 & 253 & 0.126 \\
\hline pMMR & 5 & 35 & \\
\hline dMMR & & & \\
\hline
\end{tabular}

Abbreviations:LVI.Lymphovascular invasion; PNI.Perineural invasion;MMR.mismatch repair gene; pMMR.proficient mismatch repair;dMMR.deficient mismatch repair;TDs.tumor deposits; 


\begin{tabular}{|c|c|c|c|}
\hline & TDs+ & TDs- & $\mathbf{P}$ \\
\hline pT stage & 0 & 60 & 0.000 \\
\hline T1 & 2 & 46 & \\
\hline $\mathrm{T} 2$ & 14 & 80 & \\
\hline T3 & 65 & 102 & \\
\hline \multicolumn{4}{|l|}{ T4 } \\
\hline pN stage & 2 & 102 & 0.000 \\
\hline NO & 4 & 64 & \\
\hline N1 & 14 & 51 & \\
\hline N2 & 61 & 71 & \\
\hline \multicolumn{4}{|l|}{ N3 } \\
\hline pM stage & 74 & 288 & 0.000 \\
\hline MO & 7 & 0 & \\
\hline \multicolumn{4}{|l|}{ M1 } \\
\hline clinical stage & 0 & 73 & 0.000 \\
\hline ? & 6 & 88 & \\
\hline ? & 68 & 127 & \\
\hline ૧ & 7 & 0 & \\
\hline ૧ & & & \\
\hline
\end{tabular}

2.Relationships between tumour deposits and the prognosis of gastric cancer

The TDs + group had lower DFS, CSS, and OS compared to the TDs- group(Fig. 2), and TDs + was an independent prognostic factor for DFS, CSS, and OS(Table 2). TDs $\geq 4$ had lower DFS, CSS, and OS (P< $0.05)$ (Fig. 3) .The maximum diameter of TDs was not statistically significant with prognosis $(P>0.05)$ (Fig. 4) . 
Table 2

Cox multivariate regression analysis of Disease-free survival, Cancer-specific survival, and Overall survival correlation of tumor deposits.

\begin{tabular}{|c|c|c|c|c|c|c|}
\hline & \multicolumn{2}{|l|}{ DFS } & \multicolumn{2}{|l|}{ cSS } & \multicolumn{2}{|l|}{ os } \\
\hline & $\mathrm{HR}(95 \% \mathrm{Cl})$ & $\begin{array}{l}P \\
\text { value }\end{array}$ & $\mathrm{HR}(95 \% \mathrm{Cl})$ & $\begin{array}{l}P \\
\text { value }\end{array}$ & $\mathrm{HR}(95 \% \mathrm{Cl})$ & $\begin{array}{l}\mathrm{P} \\
\text { value }\end{array}$ \\
\hline TDs & $\begin{array}{l}0.571(0.353- \\
0.923)\end{array}$ & 0.022 & $\begin{array}{l}0.464(0.302- \\
0.713)\end{array}$ & 0.000 & $\begin{array}{l}0.538(0.356- \\
0.814)\end{array}$ & 0.003 \\
\hline Age,years & - & - & - & - & $\begin{array}{l}0.638(1.089- \\
2.462)\end{array}$ & 0.018 \\
\hline Histologic grade & - & - & $\begin{array}{l}1.040(0.444- \\
2.433)\end{array}$ & 0.929 & $\begin{array}{l}1.198(0.540- \\
2.659)\end{array}$ & 0.657 \\
\hline Lauren's classification & - & - & $\begin{array}{l}0.668(0.268- \\
1.663)\end{array}$ & & $\begin{array}{l}0.579(0.248- \\
1.354)\end{array}$ & 0.446 \\
\hline Lauren(1) & & & $0.863(0.311-$ & 0.385 & $0.688(0.264-$ & $0.20 /$ \\
\hline Lauren(2) & & & $2.399)$ & 0.110 & & 0.444 \\
\hline LVI & $\begin{array}{l}0.549(0.207- \\
1.454)\end{array}$ & 0.228 & $\begin{array}{l}0.960(0.392- \\
2.348)\end{array}$ & 0.929 & $\begin{array}{l}0.940(0.404- \\
2.184)\end{array}$ & 0.885 \\
\hline PNI & $\begin{array}{l}0.511(0.259- \\
1.009)\end{array}$ & 0.053 & $\begin{array}{l}0.599(0.324- \\
1.105)\end{array}$ & 0.101 & $\begin{array}{l}0.685(0.387- \\
1.214)\end{array}$ & 0.195 \\
\hline pT stage & $\begin{array}{l}0.703(0.119- \\
4.158)\end{array}$ & & $\begin{array}{l}0.290(0.021- \\
3.940)\end{array}$ & 0.019 & $\begin{array}{l}1.023(0.162- \\
6.454)\end{array}$ & 0.009 \\
\hline $\mathrm{T}(1)$ & 0641 ( $091-$ & 0.698 & $2000(0134-$ & 0.352 & $2879(0312-$ & 0.981 \\
\hline $\mathrm{T}(2)$ & $4.519)$ & 0.655 & $29.774)$ & 0.615 & $26.583)$ & 0.351 \\
\hline $\mathrm{T}(3)$ & $\begin{array}{l}1.070(0.144- \\
7.925)\end{array}$ & 0.947 & $\begin{array}{l}4.142(0.269- \\
63.863)\end{array}$ & 0.309 & $\begin{array}{l}6.655(0.700- \\
63.284)\end{array}$ & 0.099 \\
\hline pN stage & $\begin{array}{l}0.921(0.274- \\
3.101)\end{array}$ & 0.590 & $\begin{array}{l}1.439(0.406- \\
5.106)\end{array}$ & 0.126 & $\begin{array}{l}1.312(0.404- \\
4.262)\end{array}$ & 0.078 \\
\hline$N(1)$ & & 0.895 & & 0.573 & & 0.651 \\
\hline$N(2)$ & $2.523)$ & 0.476 & $5.205)$ & 0.885 & $4.270)$ & 0.973 \\
\hline$N(3)$ & $\begin{array}{l}0.847(0.200- \\
3.586)\end{array}$ & 0.822 & $\begin{array}{l}2.117(0.476- \\
9.409)\end{array}$ & 0.325 & $\begin{array}{l}2.022(0.506- \\
8.075)\end{array}$ & 0.319 \\
\hline pM stage & - & - & - & - & - & - \\
\hline
\end{tabular}

Constant or linear-related covariate clinical stage(3) $=$ pM stage Abbreviations:LVI,Lymphovascular invasion; PNI,Perineural invasion;TDs,tumor deposits. 


\begin{tabular}{|c|c|c|c|c|c|c|}
\hline & DFS & & css & & OS & \\
\hline \multirow{3}{*}{$\begin{array}{l}\text { clinical stage } \\
\text { clinical stage(1) } \\
\text { clinical stage(2) }\end{array}$} & $\begin{array}{l}2.258(0.297- \\
17150)\end{array}$ & & $\begin{array}{l}0.608(0.037- \\
9.896)\end{array}$ & & $\begin{array}{l}0.492(0.058- \\
4.183)\end{array}$ & \\
\hline & & 0.431 & & 0.727 & & 0.516 \\
\hline & $\begin{array}{l}4.746(0.335- \\
67.150)\end{array}$ & 0.249 & $\begin{array}{l}1.029(0.037- \\
28.548)\end{array}$ & 0.986 & $\begin{array}{l}0.775(0.054- \\
11.212)\end{array}$ & 0.852 \\
\hline clinical stage(3) & $\begin{array}{l}2.698(0.095- \\
76.460)\end{array}$ & 0.561 & $\begin{array}{l}1.901(0.060- \\
60.743)\end{array}$ & 0.716 & $\begin{array}{l}1.559(0.092- \\
26.538)\end{array}$ & 0.759 \\
\hline \multicolumn{7}{|c|}{ Constant or linear-related covariate clinical stage $(3)=p M$ stage } \\
\hline
\end{tabular}

\section{Relationships between tumour deposits and TILs}

Among the 369 gastric cancer cases, 279 showed a low-to-medium TILs (75.6\%), and 90 were high TILs (24.4\%) (Fig. 5). In the TDs + group, there were 74 cases in the low-to-medium TILs and 7 cases in the high TILs. In the TDs- group, there were 205 cases in the low-to-medium TILs and 83 cases in the high TILs, The differences between the two groups were statistically significant $(P<0.05)$ (Table 3$)$.

Table 3

Correlation of tumor deposit and Tumour-Infiltrating Lymphocytes.

\begin{tabular}{|llll|}
\hline \multicolumn{4}{|c|}{ TILs } \\
& Low-medium & High & P \\
\hline TDs+ & 74 & 7 & 0.000 \\
\hline TDs- & 205 & 83 & \\
\hline TDs:tumor deposits;TILs:Tumour-Infiltrating Lymphocytes. \\
\hline
\end{tabular}

\section{Discussion}

This study analyzed the relationship between TDs and clinicopathologic characteristics and prognosis of gastric cancer. The results found that $22.0 \%$ of the 369 gastric cancer samples was $22.0 \%$, TDs was significantly associated with gender, Lymphovascular invasion, Perineural invasion, pathological TNM and clinical stages, and significant survival differences between TDs + and TDs-. TDs was an independent prognostic factor of DFS, CSS, OS of gastric cancer.Based on this basis, this topic further studies the relationship between TDs and TILs in the tumor microenvironment, and the results found that the TDs is negatively related to the TILs, suggesting that there may be a complex relationship between TDs and tumor microenvironment, TDs and TILs may interact and affect the prognosis of patients with gastric cancer. 
In 1935, Gabriel et al. first identified and reported TDs in colorectal cancer specimens, which they thought were the results of cancer cell dissemination along blood vessels [4]. The 8th edition of the AJCC/Union for International Cancer Control defines TDs as discrete tumor nodules within the lymph drainage area of the primary carcinoma without identifiable lymph node tissue or identifiable vascular or neural structure [5]. In the pN staging of colorectal cancer, the absence of regional lymph node metastasis along with the presence of TDs within the subplasma and mesenteric tissues is classified as N1c. If both regional lymph node metastasis and TDs are present, the presence of TDs has no effect on staging, and the incidence of TDs in colorectal cancer ranges from $5-45 \%$ and is associated with a poor prognosis in colorectal cancer [13-15]. Previous studies have found that TDs are present not only in colorectal cancer but also in other solid malignancies, such as gastric, bile duct, and pancreatic cancers $[2,16]$. Currently, although a few studies have shown that the presence of TDs is an independent prognostic factor for a poor prognosis in gastric cancer $[9,10]$, the mechanism of TDs formation is unclear.For colorectal cancer, the importance of TDs has been recognized and has been included in category $N$ in the 7th edition of the TNM staging system for colorectal cancer. However, in the 8th edition of the TNM staging system, TDs are considered as a metastatic lymph node in gastric cancer, which is contrary to the findings of the current study. A recent retrospective study that included 7,445 gastric cancer cases showed that the incidence of TDs ranging from 10.6-36.7\% (mean: 20.9\%) [3]. Liang studied 1,034 gastric cancer patients, of whom 240 (23.21\%) had TDs + and found that TDs were an independent prognostic factor for gastric cancer patients [2], which is similar to our findings. Therefore, the present study demonstrates that TDs is frequently observed and is an indicator of the aggressive characteristics of GC. The presence of TD is a strong and independent prognostic factor and should be incorporated into staging strategies in GC.

Regarding the study on the number, size, and prognosis of TDs.Benoit et al. found that the number of TDs $\geq 4$ had a lower DFS in rectal cancer [7]. In the present study, we investigated the relationships among the number of TDs, maximum diameter of TDs, and prognosis of gastric cancer. The results showed that the number of TDs was closely related to DFS, CSS, and OS of gastric cancer, and there was a significant difference in survival between the two groups. But in our study ,the maximum diameter of TDs was not related to prognosis,this is similar to Raul's study,suggesting that pathologists need to pay more attention to the number of TDs when observing the sections. The critical value of TDs should be verified by larger sample studies.

TILs are T lymphocytes, B lymphocytes, and NK cells that accumulate in the area of the tumour lesion and are at the forefront of the immune response and regulatory role in the tumour immune mechanism [17].Studies have shown that the antitumor immune effect of TILs is mainly cellular, on the one hand, dendritic cells present the major histocompatibility complex molecules of captured tumor neoantigens to T cells, leading to the activation of effector T cells and killing of tumor cells, which in turn secrete suppressive cytokines and have antitumor effects[18]; however, in the majority of cancer patients, the immune system cannot However, in the majority of cancer patients, the immune system fails to function effectively: it may be due to the failure of the immune system to recognize the tumor antigen and treat the tumor antigen as its own, i.e., immune tolerance; the inability of the effector T cells to infiltrate into the tumor lesion, or the suppressor (or immunosuppressive cells) in the tumor microenvironment inhibiting 
the function of effector cells [19]. In addition, the immune system, while removing tumor cells, also "reshapes" the characteristics of tumor cells to make them more malignant and more resistant to immune attack, i.e., "immune editing" [20]. Therefore, the immune system has a "double-edged sword" role in the process of tumor cell development, because TIL is a major player in tumor immunity, and there are many different subgroups of TILs, and the role of different subgroups in tumor development varies greatly, so the impact on tumor is also different.In the present study[21], TDs have been shown to be an independent prognostic factor for gastric cancer patients, and TDs + was associated with poor prognosis in gastric cancer, which is consistent with previous studies $[9,10]$. And the present study also found that TDs were negatively correlated with TILs, and TILs levels were lower in TDs(+) group and higher in TDs(-) group.From which we can conclude that in the tumor microenvironment of gastric cancer, TDs and TILs interact with each other to regulate the development of gastric cancer, thus affecting gastric cancer prognosis of patients. However, the mechanism of the interaction between TDs and TILs has not yet been elucidated, and more studies are needed to explore it in the future.

However,there is one limitation that require further discussion. the findings of this retrospective study from a single Chinese institution may not be generalizable to other settings. Therefore, these findings should be considered only for hypothesis generation and require additional validation with more extensive studies.

\section{Conclusions}

our study found that TDs were significantly negatively correlated with TILs, TDs was an Independent predictors of the prognosis of gastric cancer, provides potential biological indicators for the diagnosis and treatment of gastric cancer, and enriches the basis of anti-tumor immunotherapy.

\section{Abbreviations}

AJCC

American Joint Cancer Committee; OS:overall survival;CSS:cancer-specific survival; DFS:disease-free survival; TDs:Tumour deposits; TILs:Tumour-infiltrating lymphocytes;DFS:Disease-free survival;CSS:cancer-specific survival;OS:overall survival

\section{Declarations}

Ethics approval and consent to participate:Participants' informed consent was not required for this study because of its retrospective nature. The Medical Ethics Committee of the First Affiliated Hospital of Jinzhou Medical University approved this study and the study was conducted in accordance with the Declaration of Helsinki.

Consent for publication: Not applicable. 
Availability of data and materials:All analyses of the data have been reported in the Supporting Information File. In case any other clarification is needed, the relevant information will be made available with permission from the corresponding author.

Competing interests:The authors declare that there are no conflict of interests.

Funding: Not applicable.

Authors' contributions: Xinyue Li performed the experiments, analyzed the data, and wrote the manuscript. Jing Yang contributed to the supervision and revision in every steps.All the authors read and approved the final manuscript.

Acknowledgments:We would like to thank Editage (www.editage.cn) for English language editing.

\section{References}

1. Sung Hyuna,Ferlay Jacques,Siegel Rebecca L,Laversanne Mathieu,Soerjomataram Isabelle,Jemal Ahmedin,Bray Freddie. Global cancer statistics 2020: GLOBOCAN estimates of incidence and mortality worldwide for 36 cancers in 185 countries.[J]. CA: a cancer journal for clinicians,2021,71(3):.

2. Liang W,Liu Yuhua,Cui Jianxin,Xi Hongqing,Zhang Kecheng,Li Jiyang,Gao Yunhe,Liu Yi,Zhang Wang,Li Shaoqing,Lu Yixun,Qiao Shen,Xue Wanguo,Qiao Zhi,Chen Lin. Tumor deposit serves as a prognostic marker in gastric cancer: A propensity score-matched analysis comparing survival outcomes[J]. Cancer Medicine,2020,9(10).

3. Cristina Graham Martínez,Nikki Knijn,Marcel Verheij,Iris D Nagtegaal,Rachel S Post. Tumour deposits are a significant prognostic factor in gastric cancer - a systematic review and meta-analysis[J]. Histopathology,2019,74(6):.

4. W. B. Gabriel. A Small High-Grade Carcinoma of the Rectum with Extensive Lymphatic Spread[J]. Journal of the Royal Society of Medicine,1947,40(13).

5. Mahul B, Amin MD,Frederick L. Greene MD,Stephen B, Edge MD,Carolyn C. Compton MD, PhD,Jeffrey E, Gershenwald MD,Robert K. Brookland MD,Laura Meyer CAPM,Donna M. Gress RHIT, CTR,David R, Byrd MD,David P. Winchester MD. The Eighth Edition AJCC Cancer Staging Manual: Continuing to build a bridge from a population-based to a more "personalized" approach to cancer staging[J]. CA: A Cancer Journal for Clinicians,2017,67(2).

6. Zhu Q.,Xu B.,Li A. Predictive Value of Tumor Deposit in the Prognosis of Patients with Stage III Rectal Cancer[J]. International Journal of Radiation Oncology*Biology*Physics,2019,105(1S).

7. Benoit O,Svrcek Magali,Creavin Ben,Bouquot Morgane,Challine Alexandre,Chafai Najim,Debove Clotilde,Voron Thibault,Parc Yann,Lefevre Jeremie H. Prognostic value of tumor deposits in rectal cancer: A monocentric series of 505 patients.[J]. Journal of surgical oncology,2020. 
8. Shrestha Anup,JunLu,Chao-HuiZheng,PingLi,Jian-WeiXie,Jia-BinW,Jian-Xian Lin,Qi-Yue Chen,LongLong Cao,Mi Lin,Qian Yu,Ying-Hong Yang,Chang-Ming Huang. Prognostic significance of perigastric tumor deposits in patients with primary gastric cancer[J]. BMC Surgery,2017,17(1):.

9. Liu HChen,ZhaoqingTang,LingChen,HaojieLi,XuefeiWang,Fenglin,Yihong Sun. Evaluation of the impact of tumor deposits on prognosis in gastric cancer and a proposal for their incorporation into the AJCC staging system[J]. European Journal of Surgical Oncology,2018:

10. Mansuri N,Birkman EvaMaria,Heuser Vanina D,Lintunen Minnamaija,Ålgars Annika,Sundström Jari,Ristamäki Raija,Lehtinen Laura,Carpén Olli. Association of tumor-infiltrating T lymphocytes with intestinal-type gastric cancer molecular subtypes and outcome.[J]. Virchows Archiv: an international journal of pathology,2020:.

11. Zhang D,He Wenting,Wu Chao,Tan Yan,He Yang,Xu Bin,Chen Lujun,Li Qing,Jiang Jingting. Scoring System for Tumor-Infiltrating Lymphocytes and Its Prognostic Value for Gastric Cancer.[J]. Frontiers in immunology,2019,10.

12. Salgado R,C. Denkert,Demaria S,N. Sirtaine,Klauschen F,G. Pruneri,Wienert S,G. Van den Eynden,Baehner FL,F. Penault-Llorca,Perez EA,E.A. Thompson,Symmans WF,A.L. Richardson,Brock J,C. Criscitiello,Bailey H,M. Ignatiadis,Floris G,J. Sparano,Kos Z,T. Nielsen,Rimm DL,K.H. Allison,ReisFilho JS,S. Loibl,Sotiriou C,G. Viale,Badve S,S. Adams,Willard-Gallo K,S. Loi. The evaluation of tumorinfiltrating lymphocytes (TILs) in breast cancer: recommendations by an International TILs Working Group 2014[J]. Annals of Oncology,2015,26(2).

13. Liu Fangqi,ZhaoJiang,LiCong,WuYuchen,Song. Wang,Guo Tianan,Chen Shiqing,Cai Sanjun,Huang Dan,Xu Ye. The unique prognostic characteristics of tumor deposits in colorectal cancer patients.[J]. Annals of translational medicine,2019,7(23).

14. Raul S, Gonzalez,Justin MM, Cates,Chanjuan Shi. Number, not size, of mesenteric tumor deposits affects prognosis of small intestinal well-differentiated neuroendocrine tumors[J]. Modern Pathology: Publishing innovative clinical and translational research in the pathology of human disease,2018,31(10).

15. Basnet Shiva,Lou Qi-Feng,Liu Nan,Rana Ramesh,Shah Abilasha,Khadka Mamata,Warrier Hemanshu,Sigdel Shushil,Dhakal Sunil,Devkota Anita,Mishra Roshan,Sapkota Ganga,Zheng Liang,Ge Hai-Yan. Tumor deposit is an independent prognostic indicator in patients who underwent radical resection for colorectal cancer.[J]. Journal of Cancer,2018,9(21).

16. Fata Cynthia R,Gonzalez Raul S,Liu Eric,Cates Justin M,Shi Chanjuan. Mesenteric Tumor Deposits in Midgut Small Intestinal Neuroendocrine Tumors Are a Stronger Indicator Than Lymph Node Metastasis for Liver Metastasis and Poor Prognosis.[J]. The American journal of surgical pathology,2017,41(1).

17. Chic Nuria,Luen Stephen J,Nuciforo Paolo,Salgado Roberto,Fumagalli Debora,Hilbers Florentine,Wang Yingbo,de Azambuja Evandro,Làng István,Di Cosimo Serena,Saura Cristina,Huober Jens,Prat Aleix,Loi Sherene. Tumor Cellularity and Infiltrating Lymphocytes (CelTIL) as a Survival Surrogate in HER2-Positive Breast Cancer.[J]. Journal of the National Cancer Institute,2021:. 
18. Karolina Palucka,Lisa A, Coussens M. The Basis of Oncoimmunology[J]. Cell,2016,164(6):.

19. Greg T. Motz,George Coukos. Deciphering and Reversing Tumor Immune Suppression[J]. Immunity,2013,39(1):

20. Spranger Stefani. Mechanisms of tumor escape in the context of the T-cell-inflamed and the non-Tcell-inflamed tumor microenvironment.[J]. International immunology,2016,28(8).

21. Byung Woog Kang,Jong Gwang Kim,In Hee Lee,Han Ik Bae,An Na Seo.Clinical significance of tumorinfiltrating lymphocytes for gastric cancer in the era of immunology[J]. World Journal of Gastrointestinal Oncology,2017,9(07):293-299.

\section{Figures}




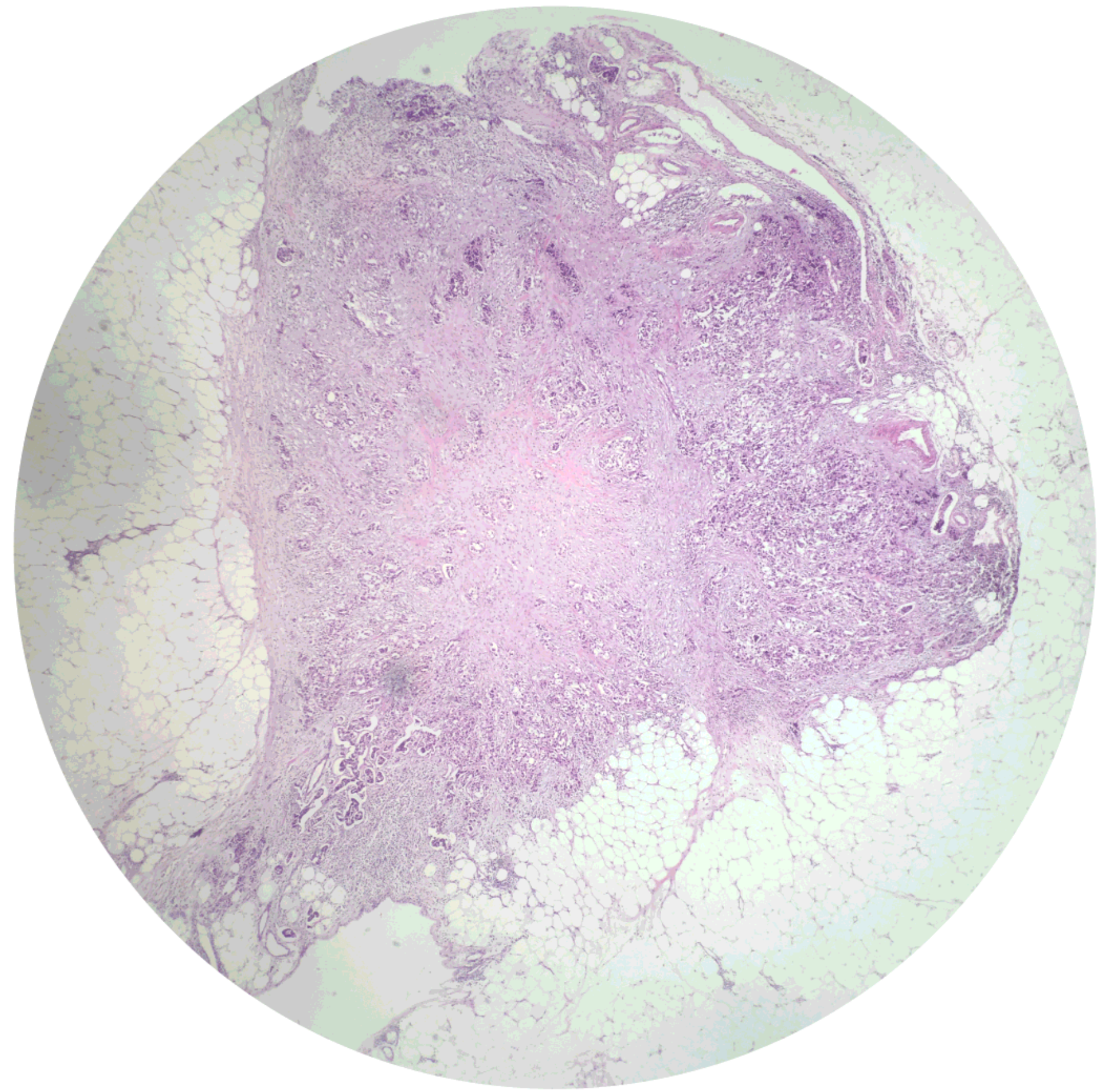

Figure 1

An example of a tumor deposit (TD) of gastric cancer to show the pathological features tumor deposits(TDs) were clearly defined as discrete tumour nodules within the lymphatic drainage area of primary cancer and without identifiable lymph node tissue or identifiable vascular or neural structures . (HE - hematoxylin and eosin $\times 10)$. 

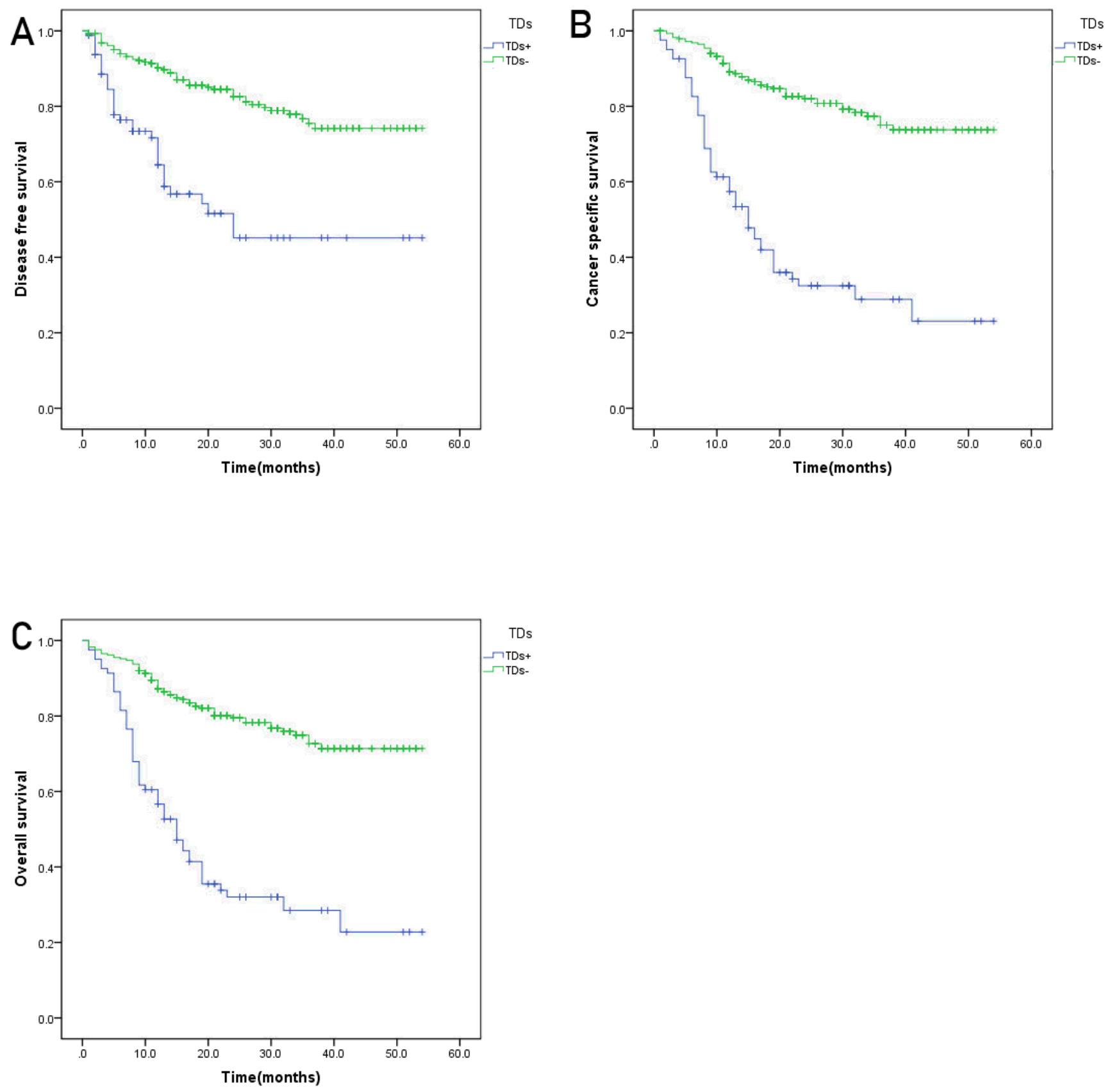

Figure 2

Kaplan-Meier curves for tumor deposits Kaplan-Meier curves for tumor deposits.A: Disease-free survival (DFS); B: Cancer-specific survival (CSS); C: Overall survival (OS).P®0.05. 

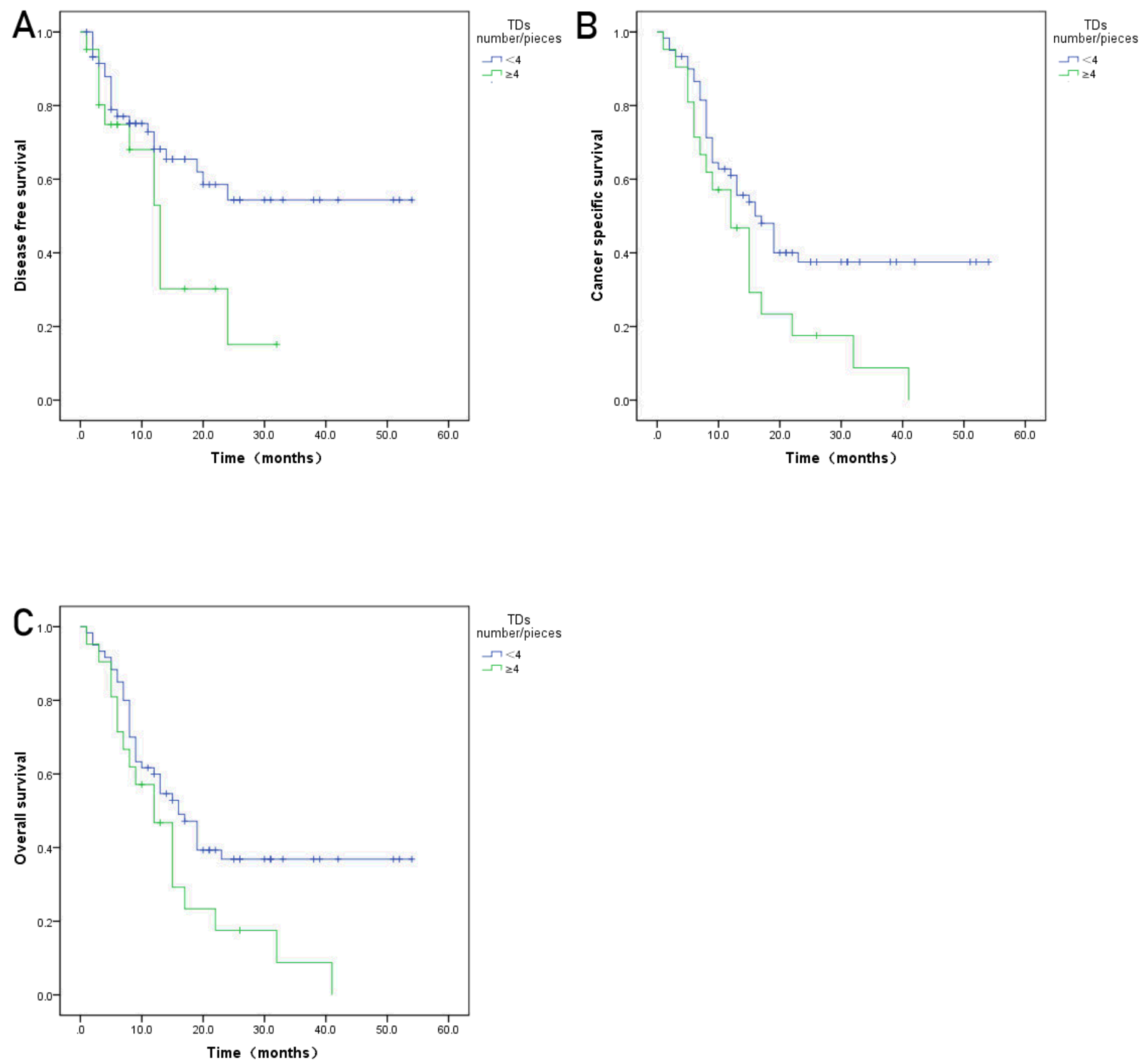

Figure 3

Kaplan-Meier curves for number of tumor deposits Kaplan-Meier curves for number of tumor deposits.A: Disease-free survival (DFS); B: Cancer-specific survival (CSS); C: Overall survival (OS).Pख0.05. 

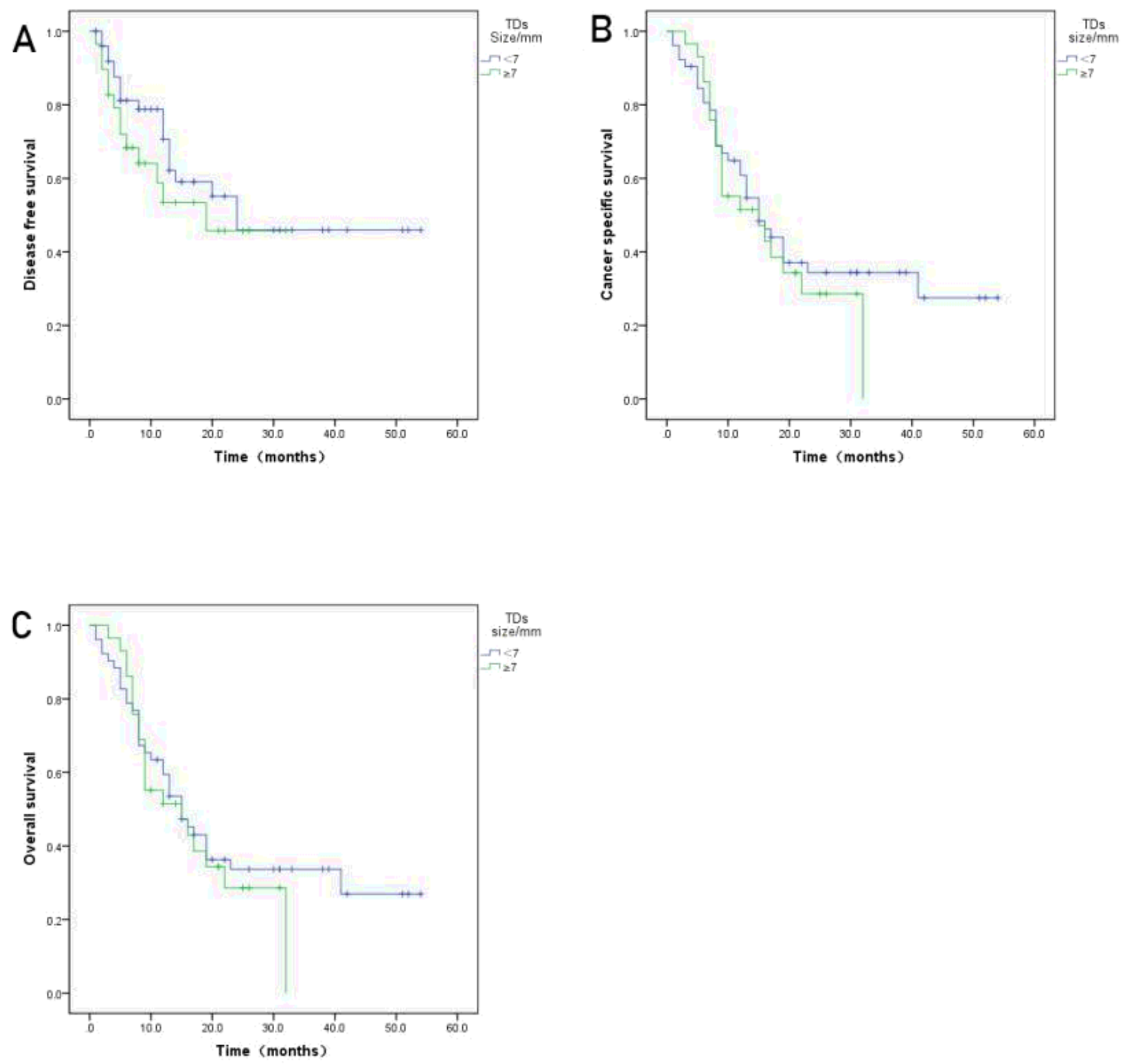

\section{Figure 4}

Kaplan-Meier curves for size of tumor deposit Kaplan-Meier curves for size of tumor deposits.A: Diseasefree survival (DFS); B: Cancer-specific survival (CSS); C: Overall survival (OS).P®0.05. 

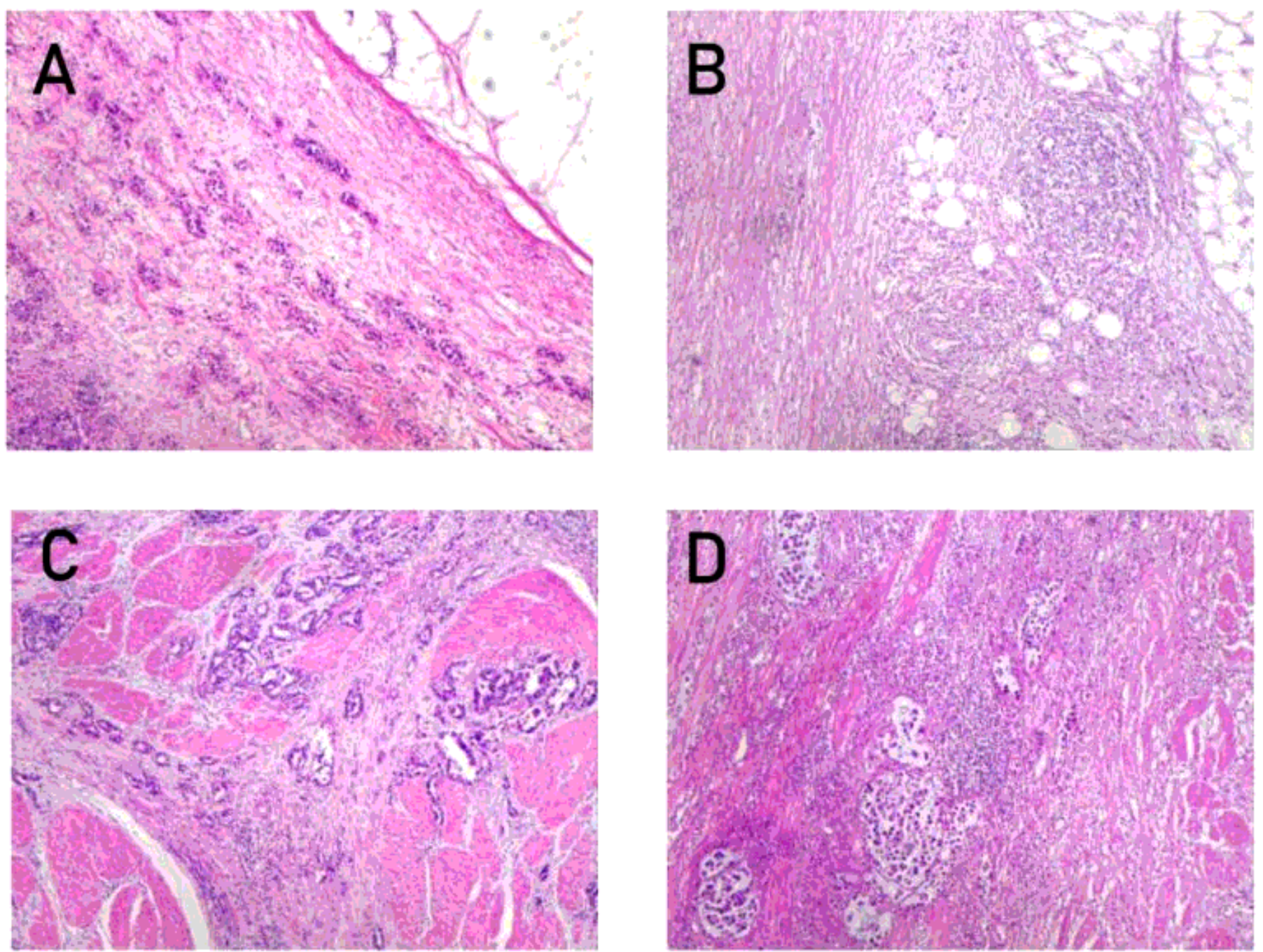

\section{Figure 5}

Expression of Gastric Cancer Tumour-Infiltrating Lymphocytes Expression of Gastric Cancer TumourInfiltrating Lymphocytes A and C: low-to-moderate Tumour-Infiltrating Lymphocytes;B and D: high Tumour-Infiltrating Lymphocytes .(HE - hematoxylin and eosin×40). 